\title{
Complications of Sepsis in Infant. A Case Report
}

DOI: $10.1515 /$ jccm-2016-0012

\author{
Lorena Elena Meliț ${ }^{1}$, Cristina Oana Mărginean²*, Anca Georgescu ${ }^{3}$, Carmen Duicu² \\ 1 Pediatrics Clinic 1, County Clinical Emergency Hospital \\ 2 University of Medicine and Pharmacy Tîrgu Mureș, Pediatrics Clinic 1 \\ 3 University of Medicine and Pharmacy Tîrgu Mureș, Infectious Diseases Clinic 1
}

\begin{abstract}
Sepsis is a systemic inflammatory response (SIRS) characterized by two or more of the following: fever $>38.5^{\circ} \mathrm{C}$ or $<36^{\circ} \mathrm{C}$, tachycardia, medium respiratory frequency over two SD for age, increased number of leukocytes.

The following is a case of an eight months old, female infant, admitted in to the clinic for fever (39.7 C), with an onset five days before the admission, following trauma to the inferior lip and gum. Other than the trauma to the lip and gum, a clinical exam did not reveal any other pathological results. The laboratory tests showed leukocytosis, positive acute phase reactants (ESR $105 \mathrm{~mm} / \mathrm{h}$, PCR $85 \mathrm{mg} / \mathrm{dl}$ ), with positive blood culture for Staphylococcus aureus MSSA. at 24 hours. Three days from admission, despite the administration of antibiotics (Vancomycin+Meronem), there was no remission of fever, and the infant developed a fluctuant collection above the knee joint. This was drained, and was of a serous macroscopic nature. A decision was made to perform a CT, which confirmed the diagnosis of septic arthritis. At two days after the intervention, the fever reappeared, therefore the antibiotic regime were altered (Oxacillin instead of Vancomycin), resulting in resolution of the fever. Sepsis in infant is a complex pathology, with non-specific symptoms and unpredictable evolution.
\end{abstract}

Keywords: sepsis, systemic inflammatory response, Staphylococcus aureus

Received: 07 February 2016 / Accepted: 10 April 2016

\section{INTRODUCTION}

The word sepsis has its origin in the Greek word 'sepo', which means decomposition due to the fact that it leads on to tissue structural and functional impairment [1,2]. In 1991, the American College of Chest Physicians (ACCP) and the Society of Critical Care Medicine (SCCM) used a new term 'Systemic Inflammatory Response Syndrome' (SIRS), which is more useful in clinical practice, and it requires at least two of the following criteria to be present: hyperthermia or hypothermia; tachypnea; tachycardia, and leukocytosis or leukopenia with immature neutrophils $[3,4]$. Thus, sepsis or SIRS represents the presence of infection associated with other general systemic signs and symptoms. All these signs and symptoms are a result of the systemic inflammatory response. Nowadays, it is clear that sepsis can be the result of bacterial, viral, fungal or parasitic infections. All these infections lead on to inflammation, and the release of various inflammatory agents and finally to cellular and organ dysfunction. Though it is often easy to arrive at a diagnosis, the etiology is in most of cases impossible to identify. The most important tests used to identify the etiology are blood cultures. The main condition, in order to increase the chances of identifying the correct etiological agent is to have a blood sample from a patient during the febrile period. Even so, most of the blood cultures came back with a negative result. In some cases, when the patient develops pleurisy or arthritis, for example, the etiological agent can be identified from the aspirated fluid. It is very important in every case to identify the etiological agent as this will aid formulating a proper and efficacious treatment plan.

The management of sepsis represents a challenge for the physician. The therapy must be initiated as quickly as possible when sepsis is suspected because it seems

\footnotetext{
Correspondence to: Cristina Oana Mărginean, University of Medicine and Pharmacy Tîrgu Mureș, 38 Gh. Marinescu Street, Tîrgu Mureș, 540139, Romania. E-mail: marginean.oana@gmail. com

Lorena Elena Meliț: Pediatrics Clinic 1, Emergency Clinical County Hospital, 38 Gh. Marinescu Street, Tîrgu Mureș, 540139, Romania.

Cristina Oana Mărginean: University of Medicine and Pharmacy Tîrgu Mureș, Pediatrics Clinic 1, 38 Gh. Marinescu Street, Tîrgu Mureș, 540139, Romania.

Anca Georgescu: University of Medicine and Pharmacy Tîrgu Mureș, Infectious Diseases Clinic 1, 38 Gh. Marinescu Street, Tîrgu Mureș, 540139, Romania.

Carmen Duicu: University of Medicine and Pharmacy Tîrgu Mureș, Pediatrics Clinic 1, 38 Gh. Marinescu Street, Tîrgu Mureș, 540139, Romania.
} 
that the first sixty minutes are the most important, being called 'the golden hour'. The mainstay of initial therapy is early aggressive treatment to restore the balance between oxygen delivery and demand, using oxygen, fluids, vasoactive drugs, antibiotics and methods to improve the ventilation [5]. Thus, the supportive treatment consists of providing an adequate oxygenation, fluid administration by vein, the correction of hydro-electrolytic and metabolic imbalances. The etiological treatment should be initiated before knowing the causing agent, guided by the patient's age, the immune status, the possibility of a nosocomial infection, the ability of the antibiotic to penetrate different tissues, and last, but not least, the acknowledgement of the organism susceptibility to different pathogenic agents. Afterwards, the treatment should be guided by the causative agent isolated from the cultures and tested on different antibiotics. The treatment of sepsis complications will depend on the affected organ and may involve a multi-disciplinary approach.

\section{CASE PRESENTATION}

The case of a 9 month-old infant admitted to the Pediatrics Clinic 1 is reported. The child had had a fever $\left(38.6{ }^{\circ} \mathrm{C}\right)$ for approximately six days. The family history did not reveal anything relevant. The personal physiological history revealed the following: the patient was the first baby, from a physiological pregnancy, born at term, vaginally, having a birth weight of $3330 \mathrm{~g}$ and APGAR score 6/1 minute and 9/5 minutes (perinatal hypoxia), she was breastfed until the age of 5 months, and after that with formula food, diversified adequately, vaccinated according to age. The personal pathological history revealed perinatal hypoxia, and a trauma due to falling approximately ten days previous to admission. The fall had resulted in a hematoma of the inferior lip and gum. The onset of fever was six days before the admission and the mother presented at the general practitioner, who recommended antipyretics and interpreted the fever as associated with tooth eruption. The fever persisted in spite of antipyretics, and instigated the mother to attend the pediatrics specialist physician, who referred her for admission to the Pediatrics Clinic 1, Emergency Clinical County Hospital Tîrgu Mureș in order to establish a diagnosis and to receive adequate treatment.

The clinical exam, on admission showed the child with pallor, warm, dry skin, presternal haematoma post-trauma, dry lips, without any other pathological elements. The patient weighted $8100 \mathrm{~g}$. The laboratory investigations showed leukocytosis with neutrophilia

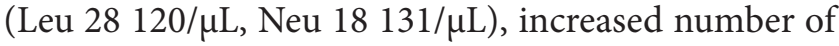
platelets $(515000 / \mu \mathrm{L})$, microcytic hypochromic anemia (Hb 8.1 g/dL, Htc 25\%, HEM 25.8 pg, MCV 79.1), increased acute phase reactants (ESR: $118 \mathrm{~mm} / \mathrm{h}, \mathrm{CRP}$ : $82.96 \mathrm{mg} / \mathrm{L}$ ), peripheral smear with signs of bacterial sepsis. Other investigations were carried out including urine examination, TORCH serology, hepatitis B and $\mathrm{C}$ serology, HIV serology, immunogram. All were in normal ranges. The level of serum cooper was increased $(214.10 \mu \mathrm{g} / \mathrm{dL})$. The culture from the blood revealed Staphylococcus aureus MSSA, with sensitivity to all the tested antibiotics. It was decided to administer Vancomycin and Meronem, but after three day of treatment, the fever persisted, and the infant developed a localized oedema of the anterior, inferior right thigh, with functional impotence, pain on mobilization and antalgic position in external rotation and semi-flexion. Therefore an ultrasound of the involved region was performed, which showed a non-homogenous, hypoechoic collection, $27 / 11 \mathrm{~mm}$ in size, without Doppler signal (Figure 1). An abdominal ultrasound was also carried out and showed the liver and spleen to be increased in size.

The CT of the right inferior limb revealed signs of synovitis in the knee joint, intraarticular and supra-patellar accumulations of fluid, with involvement of the muscular quadriceps planes, suggestive of infectious arthritis. A surgical incision and drainage of the fluid was performed. The bacteriological exam from the drained fluid showed moderately positive leukocytes, multiple erythrocytes, though culture did not reveal any bacterial growth. The fever disappeared after the incision and drainage, but after approximately forty

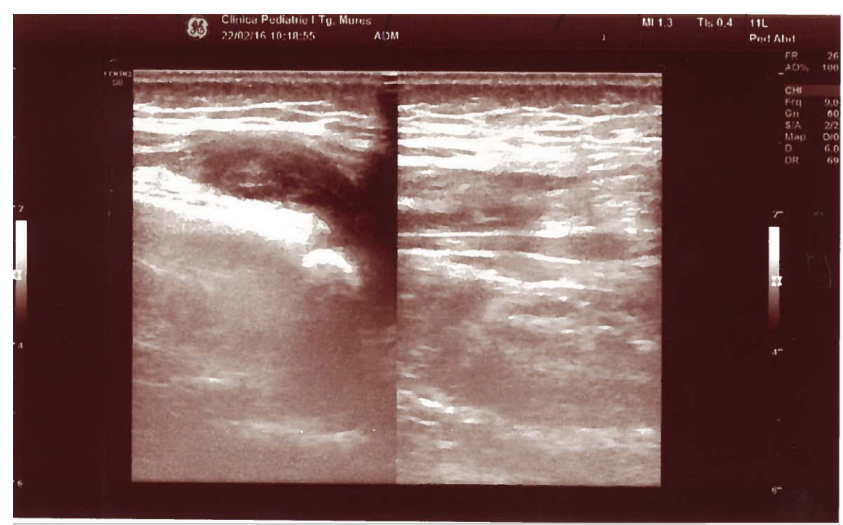

Fig. 1. Ultrasound aspect of the collection in the right thigh 
eight hours, the child developed a fever $\left(38.9^{\circ} \mathrm{C}\right)$. The infectious diseases consult recommended Oxacillin instead of Vancomycin and the administration of human immunoglobulin. Additionally, an echocardiography report to exclude endocarditis with periodical followups for early detection of a possible vegetation growth was done, with normal results. The final diagnoses were: Sepsis with Staphylococcus aureus MSSA, Septic arthritis of the right knee, Abscess of the right thigh, Anemia, secondary thrombocytosis, and enlarged liver and spleen. After the administration of Oxacillin, there was remission of fever, healing of the surgical incision and regaining of the right inferior limb mobilization. In addition to the stated antibiotic therapy, the patient also received antimycotic drugs (Diflucan), human immunoglobulin, antipyretics (Paracetamol, Algocalmin, Nurofen), protectors for the liver (Aspatofort), platelets antiaggregant therapy (Dipiridamol), probiotics (Protectis), perfusions for hydro-electrolytic rehydration. The surgical incision was cleaned and bandaged every forty eight hours. On the $28^{\text {th }}$ day of admission, the blood tests showed: Leu $13011 / \mu \mathrm{L}, \mathrm{Hb} 10.1 \mathrm{~g} / \mathrm{dL}$, Htc 30.7\%, HEM 24.8 pg, MCV $75.2 \mathrm{fL}$ with the persistence of the increased erythrocyte sedimentation rate $(40 \mathrm{~mm} / \mathrm{h})$, but normalization of the $\mathrm{C}$ reactive protein $(0.29 \mathrm{mg} / \mathrm{l})$. Twenty nine days after admission, the infant was discharged in good general status, with the recommendations to continue probiotic treatment for a month, to consult a pediatric specialist after a week, to have echocardiography periodical to excluded late onset endocarditis, and to immediately consult a pediatric specialist, if fever reoccurred.

\section{DISCUSSIONS}

Sepsis is a major health problem due to its increased morbidity and mortality and also to the increased costs involved in its management. For example, in USA, 7\% of all deaths among children are due to sepsis, while in the United Kingdom, $17 \%$ of the children admitted to an Intensive Care Unit, die [6,7]. Also, it seems that in the USA, the highest incidence of this pathology is among infants similar to the case presented above. In comparison to the present study, where the patient was a girl, the diagnosis of sepsis most frequently occurs in boys [6]. The children in the study had an underlying disease, whereas in the current report, no other associated pathology was identified except for perinatal hypoxia without further sequelae.
The most commonly involved sites of infection are the respiratory tract infections (37\%) and primary bloodstream infections (25\%) [6], the latter one being also characteristic for our patient. The hospitalization period described in the literature is on average thirty one days, similarly with the current case, where the infant was discharged after twenty eight days. The diagnosis of sepsis in children is often hard to establish because the clinical picture is very different from that of the adult. Even though the acute-phase reactants are usually positive, there is no specific test for sepsis, therefore it is recommended that the physician use the signs and symptoms encountered at the clinical exam to establish the diagnosis of sepsis and septic shock. The management of sepsis includes supportive treatment, and antimicrobial therapy, which initially is empiric, targeting the probable causative agents depending on the host's factors (age, immune status), the factors of the pathogenic agent, and antibiotic properties [8]. After the isolation of the causative agent, the antibiotic regimen should be administered according to the sensitivity in order to avoid multidrug-resistance [9]. Initially a combination of two wide-spectrum antibiotics (Vancomycin with Meronem) was prescribed due to the patient's age and because the primary site of infection was not identified initially. Because of the difficulty in identifying the causative agent, broad-spectrum antibiotics should be used with the aim to control the infection and prevent the complications. Once the causative agent had been identified the initial antibiotic regime was continued because Staphylococcus aureus was sensitive to Vancomycin. The studies underline that sometimes it is better to administer Vancomycin due to the fact that even community-acquired Staphylococcus aureus is often resistant to other types of antibiotics [10]. Vancomycin was initially prescribed for Staphylococcus aureus MSSA isolated from the blood culture, but despite this, the progress was unfavorable until Vancomycin was changed with Oxacillin. In comparison to other pathology, in sepsis, supportive treatment is as important as the antimicrobial therapy. The balance between procoagulant and anticoagulant factors is also impaired in sepsis, with an increase in procoagulant factors and a decrease of anticoagulant ones [11]. Due to this fact and also due to the increased number of platelets, it was decided to introduce platelet antiaggregant therapy (Dipiridamol). The normal function of the liver is often impaired during the evolution of sepsis due to both, the pathology itself and the sideeffects of the administered therapy, therefore it was de- 
cided to administer liver protectors. Even though studies show only a slight improvement in the development of sepsis after the administering immunoglobulin [12], it was decided to implement this therapy, considering the age of the patient. The complications of sepsis are very diverse and severe involving almost every organ. Infectious arthritis and acute osteomyelitis in children are usually a result of haematogenous bacterial spread, and the most frequent the causative agent is Staphylococcus aureus (80\%), though others such as Streptococcus pneumoniae and Haemophilus influenza can be involved [13-15]. In one study, Staphylococcus aureus was the causative organism in 54\% of the cases of osteomyelitis and septic arthritis [13]. Also, similarly with the case presented here, the most affected part of the body, was the lower limb [13]. The function assessment at the follow-up was normal in $91 \%$ of the studied cases. In the case presented above, the infant regained the function of the lower limb completely.

\section{- CONCLUSIONS}

This case repot highlights the need to investigate and adequately diagnose and treat all febrile illnesses to avoid the development of sepsis.

\section{CONFLICT OF INTEREST}

Nothing to declare

\section{REFERENCES}

1. Geroulanos S, Douka ET. Historical perspective of the word 'sepsis'. Intensive Care Med. 2006;32:2077.

2. Vincent JL, Abraham E .The last 100 years of sepsis. Am J Respir
Crit Care Med. 2006;173:256-63.

3. Poeze M, Ramsay G, Gerlach H, et al. An international sepsis survey: a study of doctor's knowledge and perception about sepsis. Crit Care. 2004;8:R409-13.

4. Bone RC, Grodzin CJ, Balk RA. Sepsis: a new hypothesis for pathogenesis of the disease process. Chest. 1997;112:235-43.

5. Rivers E, Nguyen B, Havstad S, et al. Early goal-directed therapy in the treatment of severe sepsis and septic shock. $N$ Engl J Med. 2001;345(19):1368-77.

6. Watson RS, Carcillo JA, Linde-Zwirble WT, et al. The epidemiology of severe sepsis in children in the United States. Am J Respir Crit Care Med. 2003;167:695-701.

7. Inwald DP, Tasker RC, Peters MJ, Nadel S. Emergency management of children with severe sepsisin the United Kingdom: the results of the Pediatric Intensive Care Society sepsis audit. Arch Dis Child. 2009;94:348-53.

8. Kumar A, Ellis P, Arabi $Y$ et al. Initiation of inappropriate antimicrobial therapy results in a fivefold reduction of survival in human septic shock. Chest. 2009;136:1237-48.

9. Simon N. Severe pediatric sepsis. Expert Rev Anti Infect Ther. 2012;10:111-4.

10. Kaplan SL, Hulten KG, Gonzalez BE et al. Three-year surveillance of community-acquired Staphylococcus aureus in children. Clin Infect Dis. 2005;40:1785-91.

11. Russel JA. Management of sepsis. N Engl J Med. 2006;355:1699713.

12. Werdan K, Pilz G, Bujdoso O et al. Score-based immunoglobulin $\mathrm{G}$ therapy of patients with sepsis: The SBITS study. Crit Care Med. 2007;35:2693-701.

13. Brischetto A, Leung G, Marshall C, Bowen A. A retrospective Case-Series of Children With Bone and Joint Infection From Northern Australia. Medicine. 2016;95:e2885.

14. Pääkkökknen M, Peltola H. Acute osteomyelitis in children. N Engl J Med. 2014;370:1365-66.

15. Street M, Puna R, Huang M, et al. Pediatric acute hematogenous osteomyelitis. J Pediatr Orthop. 2015;35:634-39. 